\title{
Ionization Efficiency in the Dayside Martian Upper Atmosphere
}

\author{
J. Cui ${ }^{1,2,3}$ (1), X.-S. Wu ${ }^{2}$, S.-S. Xu ${ }^{4}$, X.-D. Wang ${ }^{5}$, A. Wellbrock ${ }^{6,7}$, T. A. Nordheim ${ }^{8}$, Y.-T. Cao ${ }^{2}$, \\ W.-R. Wang ${ }^{2}$, W.-Q. Sun ${ }^{3}$, S.-Q. Wu ${ }^{9}$, and Y. Wei ${ }^{10}$ \\ ${ }^{1}$ School of Atmospheric Sciences, Sun Yat-sen University, Zhuhai, Guangdong, People's Republic of China \\ ${ }^{2}$ National Astronomical Observatories, Chinese Academy of Sciences, Beijing, People's Republic of China \\ ${ }^{3}$ Lunar and Planetary Science Laboratory, Macau University of Science and Technology, Macau, People's Republic of China \\ ${ }^{4}$ Space Science Laboratory, University of California, Berkeley, CA, USA \\ ${ }^{5}$ Swedish Institute of Space Physics, Kiruna, Sweden \\ ${ }^{6}$ Mullard Space Science Laboratory, University College London, Dorking, UK \\ ${ }^{7}$ The Centre for Planetary Sciences at University College London/Birkbeck, London, UK \\ 8 Jet Propulsion Laboratory, California Institute of Technology, Pasadena, CA, USA \\ ${ }^{9}$ School of Space and Environment, Beihang University, Beijing, People's Republic of China \\ ${ }^{10}$ Institute of Geology and Geophysics, Chinese Academy of Sciences, Beijing, People's Republic of China \\ Received 2018 March 15; revised 2018 April 8; accepted 2018 April 9; published 2018 April 20
}

\begin{abstract}
Combining the Mars Atmosphere and Volatile Evolution measurements of neutral atmospheric density, solar EUV/X-ray flux, and differential photoelectron intensity made during 240 nominal orbits, we calculate the ionization efficiency, defined as the ratio of the secondary (photoelectron impact) ionization rate to the primary (photon impact) ionization rate, in the dayside Martian upper atmosphere under a range of solar illumination conditions. Both the $\mathrm{CO}_{2}$ and $\mathrm{O}$ ionization efficiencies tend to be constant from $160 \mathrm{~km}$ up to $250 \mathrm{~km}$, with respective median values of $0.19 \pm 0.03$ and $0.27 \pm 0.04$. These values are useful for fast calculation of the ionization rate in the dayside Martian upper atmosphere, without the need to construct photoelectron transport models. No substantial diurnal and solar cycle variations can be identified, except for a marginal trend of reduced ionization efficiency approaching the terminator. These observations are favorably interpreted by a simple scenario with ionization efficiencies, as a first approximation, determined by a comparison between relevant cross sections. Our analysis further reveals a connection between regions with strong crustal magnetic fields and regions with high ionization efficiencies, which are likely indicative of more efficient vertical transport of photoelectrons near magnetic anomalies.
\end{abstract}

Key words: planets and satellites: atmospheres - planets and satellites: individual (Mars)

\section{Introduction}

The Martian ionosphere, the ionized portion of the Martian upper atmosphere, contains at the dayside a well-defined primary layer and a low altitude secondary layer, which are produced by solar EUV/X-ray ionization along with impact ionization by photoelectrons (Withers 2009, and references therein). These processes are parameterized by the primary and secondary ionization rates, respectively, with the ratio of the latter to the former being frequently termed as ionization efficiency (Richards \& Torr 1988). The calculation of the primary ionization rate is straightforward with the aid of the classical Beer-Lambert law, whereas the calculation of the secondary ionization rate, which requires either the implementation of the Monte Carlo algorithm (e.g., Bhardwaj \& Jain 2009) or the multi-stream solution to the Boltzmann equation (e.g., Wedlund et al. 2011), is far more involved.

The ionization efficiency in the dayside upper atmosphere was calculated for the Earth (Lilensten et al. 1989), Titan (Lilensten et al. 2005), Mars (Nicholson et al. 2009), and Saturn (Galand et al. 2009), as well as giant exoplanets such as HD 209458b (Ionov et al. 2014). In each of the aforementioned works, a photoelectron transport model was coupled with a neutral background atmosphere model to compare the primary and secondary ionization rates under a range of solar illumination conditions. Empirical formulae for these modelbased ionization efficiencies were provided in some of the existing works, allowing fast calculation of the total ionization rates in the dayside ionospheres of selected planetary bodies.
Since information on ionization efficiency is crucial for aeronomical studies, it is instructive to validate the model results of this key parameter with real data. This was not feasible for Mars until the arrival of the Mars Atmosphere and Volatile Evolution (MAVEN) mission (Jakosky et al. 2015), which provides a unique opportunity to explore a wide parameter space of controlling factors required for calculating both primary and secondary ionization rates in the dayside Martian upper atmosphere. These controlling factors include solar EUV/X-ray flux, measured by the Extreme Ultraviolet Monitor (EUVM; Eparvier et al. 2015), the neutral atmospheric density, measured by the Neutral Gas and Ion Mass Spectrometer (NGIMS; Mahaffy et al. 2015b), as well as the differential electron intensity, measured by the Solar Wind Electron Analyzer (SWEA; Mitchell et al. 2016). The above sources of data, publicly available at the MAVEN Science Data Center (https://lasp.colorado.edu/maven/sdc/public/), are utilized in this study to determine the ionization efficiency, which is then compared with existing model results (e.g., Fox \& Yeager 2006; Haider et al. 2006; Nicholson et al. 2009). The present study is complementary to various modeling efforts made to understand the photoelectron energy spectrum observed near Mars (e.g., Sakai et al. 2015; Xu et al. 2015; Xu \& Liemohn 2015; Peterson et al. 2016), but with a special emphasis on secondary ionization as an important effect of photoelectron production. The results presented here are useful for fast calculations of the ionization rate in 
the dayside Martian upper atmosphere, without the need to construct photoelectron transport models.

\section{Calculation of Ionization Efficiency}

The primary (photon impact) and secondary (photoelectron impact) ionization rates, denoted as $P_{1}$ and $P_{2}$, are calculated via

$$
P_{1, \mathrm{j}}(z, \theta)=\sum_{\mathrm{k}} \int_{\lambda<\bar{\lambda}_{\mathrm{k}, \mathrm{j}}} \sigma_{\mathrm{k}, \mathrm{j}}^{(\mathrm{pi})}(\lambda) N_{\mathrm{j}}(z, \theta) F_{\infty}(\lambda) e^{-\tau(\lambda, z, \theta)} d \lambda,
$$

and

$$
P_{2, \mathrm{j}}(z, \theta)=\sum_{\mathrm{k}} \int_{E>\bar{E}_{\mathrm{k}, \mathrm{j}}} \sigma_{\mathrm{k}, \mathrm{j}}^{(\mathrm{e})}(E) N_{\mathrm{j}}(z, \theta) 4 \pi I_{\mathrm{e}}(E, z, \theta) d E,
$$

where $z$ is the altitude, $\theta$ is the solar zenith angle (SZA), $\lambda$ is the photon wavelength, $E$ is the electron energy, $N_{\mathrm{j}}$ is the number density of neutral species $\mathrm{j}, \sigma_{\mathrm{k}, \mathrm{j}}^{(\mathrm{pi})}$ and $\sigma_{\mathrm{k}, \mathrm{j}}^{(\mathrm{ei})}$ are the photoionization and electron impact ionization cross sections for channel $\mathrm{k}$, $\bar{\lambda}_{\mathrm{k}, \mathrm{j}}$ and $\bar{E}_{\mathrm{k}, \mathrm{j}}$ are the maximum photon wavelength and minimum electron energy required by ionization, $F_{\infty}$ is the solar EUV/X-ray flux at the top of the atmosphere, $I_{\mathrm{e}}$ is the mean differential electron intensity, $\tau$ is the line-of-sight optical depth given by

$$
\tau(\lambda, z, \theta)=\sum_{\mathrm{k}, \mathrm{j}} \sigma_{\mathrm{k}, \mathrm{j}}^{(\mathrm{pa})}(\lambda) \int_{z}^{\infty} \frac{N_{\mathrm{j}}\left(z^{\prime}, \theta\right)}{C_{\mathrm{h}}\left(z^{\prime}, \theta\right)} d z^{\prime},
$$

with $\sigma_{\mathrm{k}, \mathrm{j}}^{(\mathrm{pa})}$ being the photoabsorption cross section, and $C_{\mathrm{h}}$ is the Chapman grazing incidence function that takes into full account the spherical nature of the atmosphere (Smith \& Smith 1972). The summation in Equation (3) is over $\mathrm{CO}_{2}$ and $\mathrm{O}$, the two most abundant neutral species in the Martian upper atmosphere (Mahaffy et al. 2015a). The respective crosssection data, $\sigma_{\mathrm{k}, \mathrm{j}}^{(\mathrm{p})}, \sigma_{\mathrm{k}, \mathrm{j}}^{(\mathrm{ei})}$, and $\sigma_{\mathrm{k}, \mathrm{j}}^{(\mathrm{pa})}$, are based on our previous compilation in Cui et al. (2011).

The calculation of the primary and secondary ionization rates using Equations (1)-(3) is performed on an orbit-to-orbit basis. We include the relevant data acquired at the dayside of Mars, defined as periapsis SZA below $85^{\circ}$, during all orbits available at the time when this manuscript was being prepared, from the arrival of MAVEN at Mars on 2014 September 21 up to 2017 August 15. Only the inbound portion of each MAVEN orbit is analyzed to reduce the uncertainty in atmospheric neutral density due to contamination by possible reactions on the NGIMS antechamber walls, a well-known feature that also occurs on other mass spectrometers with a similar design (e.g., Cui et al. 2009). Both the primary and secondary ionization rates are proportional to neutral density (see Equation (2)), but this dependence cancels out when the ionization efficiency is evaluated as the ratio of the two rates. However, the primary ionization rate also depends on neutral density via the extinction in solar irradiance (see Equation (3)), implying that the uncertainty induced by wall chemistry is only a concern at locations with large extinction.

The Deep Dip (DD) campaign on 2015 April 17-22, which provides the only measurements made at the dayside and near the main ionospheric peak, is excluded in the present study. These measurements were recently used to examine the ionization balance (Mendillo et al. 2017) and thermal structure (Cui et al. 2018) in the Martian upper atmosphere. The line-ofsight optical depth for photoabsorption at EUV/X-ray is typically smaller than unity for a nominal MAVEN orbit, with extinction not exceeding $5 \%$ near the periapsis as long as the portion of the orbit below $250 \mathrm{~km}$ remains sunward of $60^{\circ}$. However, this is not the case for a typical DD orbit. Taking as an example orbit No. 1063 on 2015 April 17 with a periapsis altitude of $134 \mathrm{~km}$, the input solar energy flux at the top of the Martian atmosphere is found to be $1.5 \mathrm{erg} \mathrm{cm}^{-2} \mathrm{~s}^{-1}$ when integrated over the wavelength range of $0.1-190 \mathrm{~nm}$, but this flux declines by $40 \%$ at periapsis due to atmospheric absorption. Here the topside energy flux is found by integrating the appropriate level 3 solar spectral model at Mars, constructed from the Flare Irradiance Spectral Model-Mars and calibrated with the EUVM band irradiance data (Thiemann et al. 2017).

The SWEA instrument is capable of measuring differential electron intensity over the energy range of $3 \mathrm{eV}$ to $4.6 \mathrm{keV}$, and the signal-to-noise ratio remains above 3 up to $1 \mathrm{keV}$ for a typical electron energy spectrum (Peterson et al. 2016). Each spectrum is corrected for spacecraft charging with appropriate shift in energy using the potential measured by the Langmuir Probe and Waves (LPW; Andersson et al. 2015). The electron energy range above $13.8 \mathrm{eV}$, which is the nominal ionization potential of $\mathrm{CO}_{2}$, is crucial for calculating the secondary ionization rate in the Martian upper atmosphere (Cui et al. 2011). Therefore, each electron energy spectrum with simultaneous LPW-based potential less negative than $-10.8 \mathrm{eV}$ is excluded since its low energy portion, which is important for secondary ionization, was not sampled by the SWEA.

For illustrative purposes, two examples of the neutral densities (left), the primary (solid) and secondary (dashed) ionization rates (middle), as well as the respective ionization efficiencies (right) are shown in Figure 1, all as a function of altitude. Blue and red stand for $\mathrm{CO}_{2}$ and $\mathrm{O}$, respectively. The upper panels are for orbit No. 3043 on 2016 April 22, with a periapsis altitude of $164 \mathrm{~km}$ and an SZA of $52^{\circ}$ at northern mid-latitudes. We find for this orbit a solar EUV/X-ray energy input of $1.2 \mathrm{erg} \mathrm{cm}^{-2} \mathrm{~s}^{-1}$ at the top of the atmosphere when integrated from 0.1 to $190 \mathrm{~nm}$. The lower panels are for orbit No. 849 on 2015 March 8, with a periapsis altitude of $159 \mathrm{~km}$ and a SZA of $74^{\circ}$ at northern low-latitudes characterized by a relatively large solar energy input of $1.8 \mathrm{erg} \mathrm{cm}^{-2} \mathrm{~s}^{-1}$.

For the first example, both the $\mathrm{CO}_{2}$ and $\mathrm{O}$ ionization efficiencies tend to be fairly constant around $20 \%$ over the altitude range displayed in the figure. In contrast, the second example reveals considerable fluctuations in ionization efficiency above $190 \mathrm{~km}$, which we find to be directly related to fluctuations in the electron energy spectrum. Fluctuations in neutral atmospheric density, indicative of gravity waves (e.g., England et al. 2017), are irrelevant since they manifest in both the primary and secondary ionization rates but not in the respective ratio. A careful examination of orbit No. 849, as well as other orbits showing a similar behavior, suggests that the observed fluctuations in the electron energy spectrum are not caused by the variations in spacecraft potential either, and likely reflect a physical phenomenon. We speculate that these fluctuations are induced by Solar Wind (SW) interactions with the Martian upper atmosphere, which would then imply a substantial energy input in addition to solar radiation. This is especially a concern for orbit No. 849 since an Interplanetary Coronal Mass Ejection (ICME) was observed during this orbit (Curry et al. 2015; Dong et al. 2015). According to the model results of Sakai et al. (2015), invoking a population of external 
No. 3043 (22 Apr 2016)
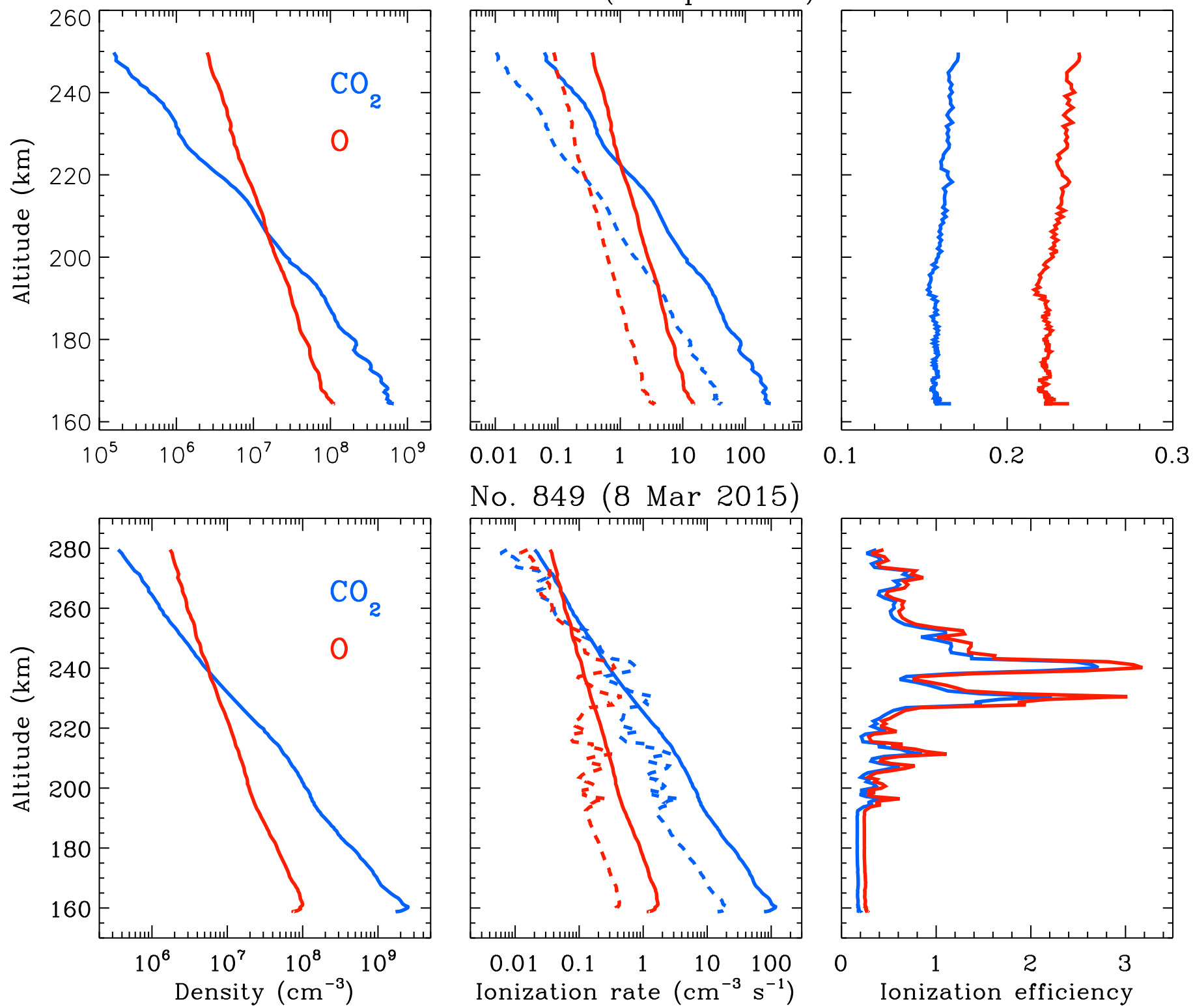

No. 849 (8 Mar 2015)
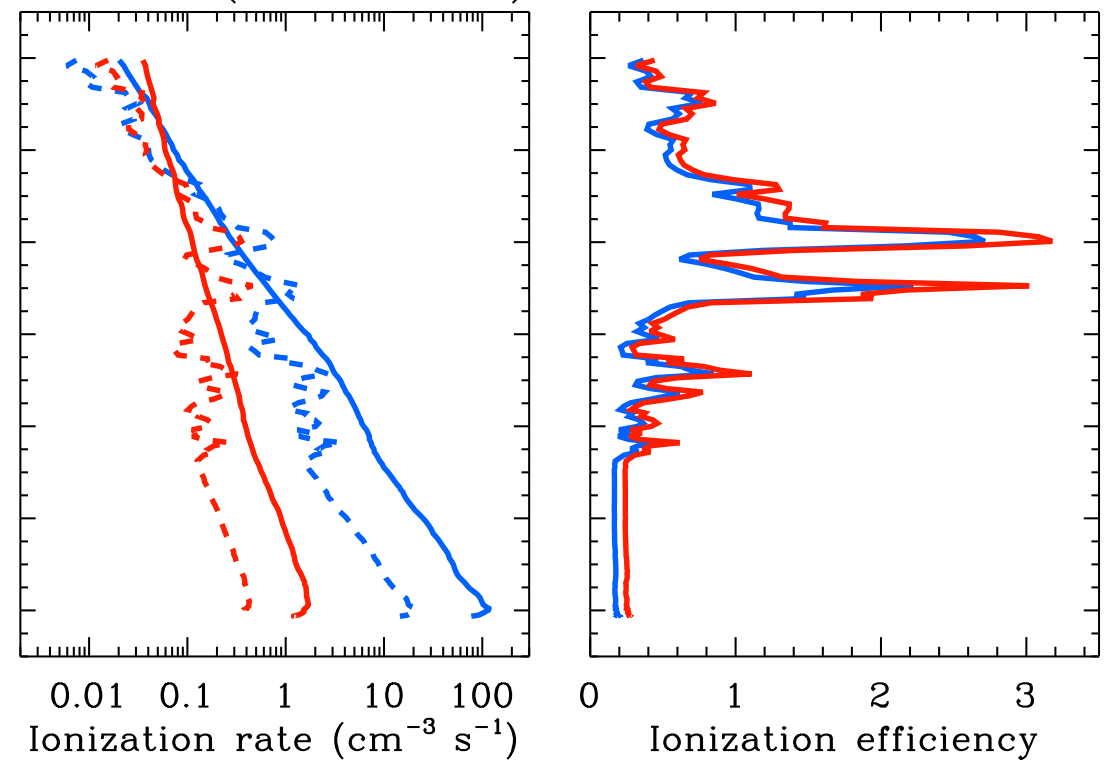

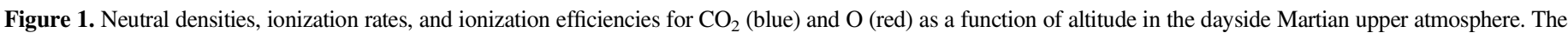

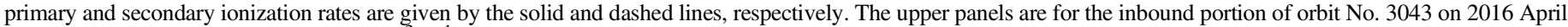

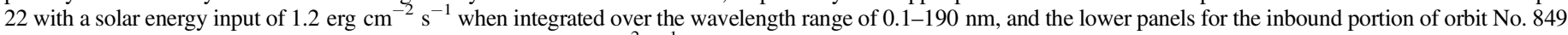

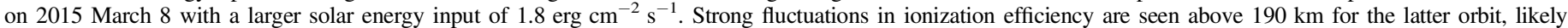

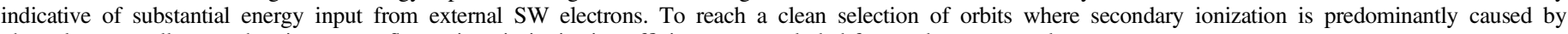
photoelectrons, all cases showing strong fluctuations in ionization efficiency are excluded from subsequent analyses.

$\mathrm{SW}$ electrons is sometimes required to interpret the electron energy spectrum observed at the dayside of Mars, a situation that is beyond the scope of this study. To be conservative, we exclude all orbits displaying strong fluctuations in ionization efficiency. This leaves a clean selection of orbits where secondary ionization is predominantly caused by photoelectrons. The exclusion of "contaminated" orbits is implemented by requiring that the relative amplitude in ionization efficiency does not exceed 5\%, where the relative amplitude is determined following our previous procedure used for calculating the wave amplitude in Titan's upper atmosphere (Cui et al. 2013, 2014). These orbits are evenly distributed in longitude but concentrated around the equator, without evidence for clustering near strong magnetic anomalies (Acuna et al. 1999). With such a data filtering process, only those orbits with smoothly varying ionization efficiencies are retained. Eventually, the data from 240 nominal MAVEN orbits are used for subsequent analysis.

\section{Magnitudes and Trends of Ionization Efficiency}

The altitude profiles of $\mathrm{CO}_{2}$ (left) and $\mathrm{O}$ (right) ionization efficiencies for all the 240 nominal MAVEN orbits are shown in Figure 2, with the red lines giving the respective median profiles along with standard deviations within the predefined altitude bins. Both ionization efficiencies remain roughly constant above $160 \mathrm{~km}$, with median values of $0.19 \pm 0.03$ for $\mathrm{CO}_{2}$ and $0.27 \pm 0.04$ for $\mathrm{O}$. For comparison, the model results of Nicholson et al. (2009), shown with the blue lines in Figure 2 and appropriate for a median SZA of $55^{\circ}$, predict 

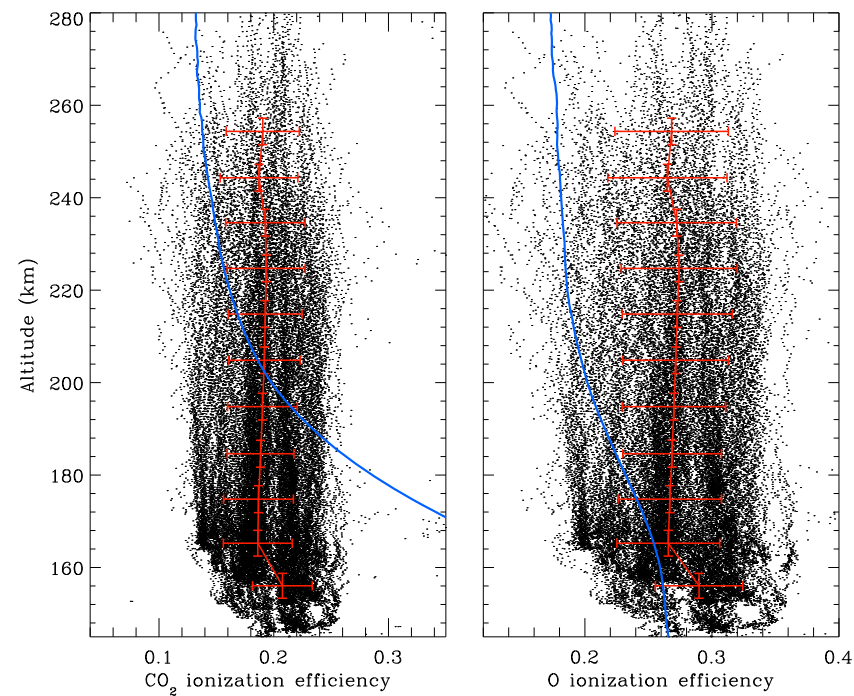

Figure 2. Altitude profiles of $\mathrm{CO}_{2}$ (left) and $\mathrm{O}$ (right) ionization efficiencies for the 240 nominal MAVEN orbits analyzed in this study. The red lines give the respective mean profiles along with standard deviations within the predefined altitude bins. For comparison, the model $\mathrm{CO}_{2}$ and $\mathrm{O}$ ionization efficiencies from Nicholson et al. (2009) are superimposed with the blue lines, appropriate for a median SZA of $55^{\circ}$

asymptotic ionization efficiencies of 0.13 for $\mathrm{CO}_{2}$ and 0.17 for O well above $200 \mathrm{~km}$. These efficiencies underestimate the $M A V E N$ results by no more than $35 \%$. At a representative altitude of $200 \mathrm{~km}$ and for an SZA of $80^{\circ}$, Haider et al. (2006) obtained primary and secondary ionization rates of $13 \mathrm{~cm}^{-3} \mathrm{~s}^{-1}$ and $2.8 \mathrm{~cm}^{-3} \mathrm{~s}^{-1}$ for $\mathrm{CO}_{2}, 2.4 \mathrm{~cm}^{-3} \mathrm{~s}^{-1}$ and $1.1 \mathrm{~cm}^{-3} \mathrm{~s}^{-1}$ for $\mathrm{O}$, respectively. These ionization rates imply a $\mathrm{CO}_{2}$ ionization efficiency of 0.22 , in agreement with the $M A V E N$ result, but a relatively high $\mathrm{O}$ ionization efficiency of 0.46 . Fox \& Yeager (2006) reported an efficiency of 0.18 at $200 \mathrm{~km}$ for total ionization, appropriate for an SZA of $60^{\circ}$. This should be compared to the $M A V E N$-based $\mathrm{CO}_{2}$ ionization efficiency of 0.19 as $\mathrm{CO}_{2}$ is the most abundant species at this altitude (Mahaffy et al. 2015a). The above comparisons indicate that a fair data-model agreement could be achieved in terms of the magnitude of the ionization efficiency at the top of the atmosphere. Modest differences present at certain cases are not unexpected since multi-stream transport models do not always reproduce satisfactorily the observed differential electron intensity in planetary ionospheres, in terms of both amplitude and shape (e.g., Cui et al. 2011; Sakai et al. 2015). In practice, fine tuning of input parameters, such as the solar $\mathrm{EUV} / \mathrm{X}$-ray irradiance, is required to reduce the data-model disagreement to a level below the combined instrument uncertainty (e.g., Peterson et al. 2016).

In terms of the shape of the ionization efficiency, Figure 2 reveals a clear distinction between the MAVEN measurements and the model calculations of Nicholson et al. (2009). The latter predicted a steep gradient in $\mathrm{CO}_{2}$ ionization efficiency with an enhancement by more than a factor of 2 from $200 \mathrm{~km}$ down to $160 \mathrm{~km}$. An enhancement in $\mathrm{O}$ ionization efficiency is predicted by the same modeling work, though to a lesser extent. These features are to be distinguished from the MAVEN results that indicate no discernible variations in both ionization efficiencies down to $160 \mathrm{~km}$. We caution that the critical altitude below which the ionization efficiency no longer remains constant varies substantially between different models. For instance, Fox \& Yeager (2006) reported a constant total
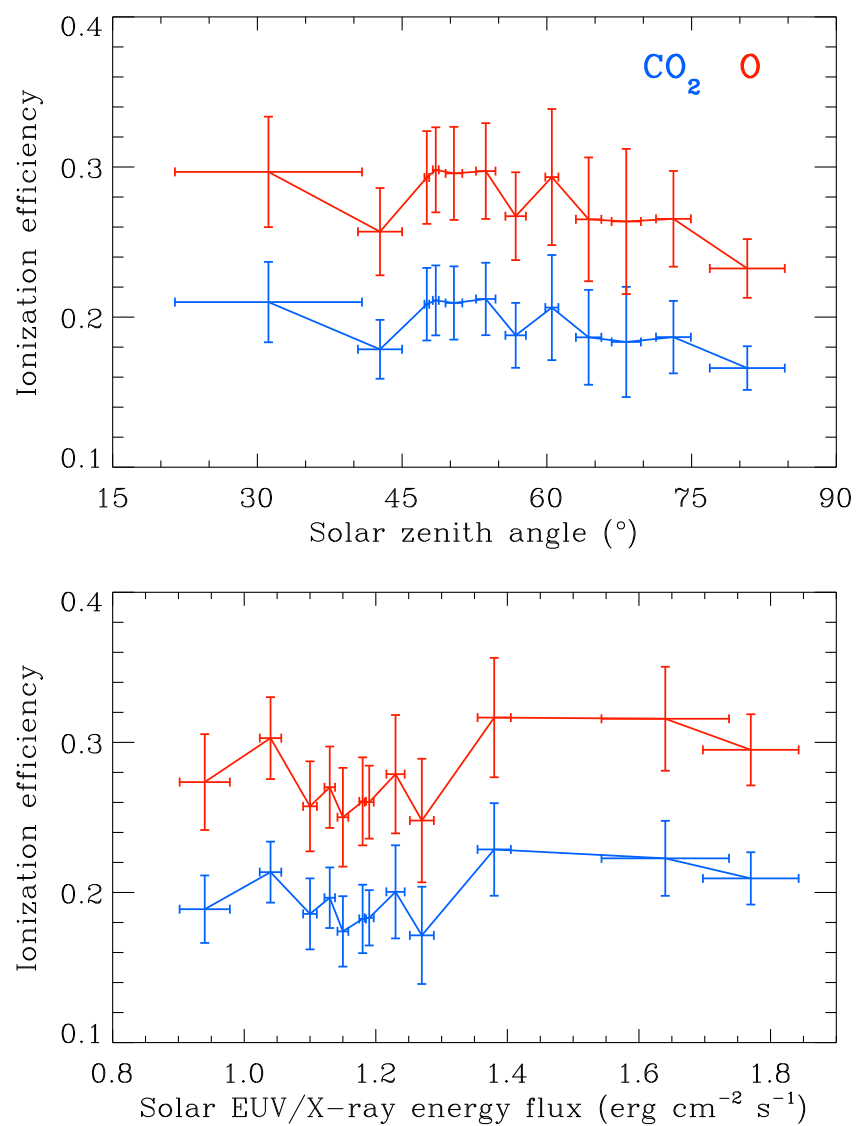

Figure 3. Diurnal (top) and solar cycle (bottom) variations of $\mathrm{CO}_{2}$ (blue) and $\mathrm{O}$ (red) ionization efficiencies at the top of the dayside Martian upper atmosphere based on the MAVEN data acquired during the selected 240 nominal orbits.

ionization efficiency of 0.18 from the top of the atmosphere down to at least $160 \mathrm{~km}$, consistent with the MAVEN measurements shown in Figure 2. Below $160 \mathrm{~km}$, the $M A V E N$-based $\mathrm{CO}_{2}$ and $\mathrm{O}$ ionization efficiencies rise by $10 \%$ over a vertical extent of $10 \mathrm{~km}$. However, such an observation deserves some caution since it is unclear whether this is a real phenomenon or a mere representation of enhanced wall chemistry near periapsis. Specifically, the effect of wall chemistry overestimates the neutral density as well as the line-of-sight optical depth, which implies that the primary ionization rate is underestimated and thus the ionization efficiency overestimated. Both a rigorous treatment of chemical reactions on the NGIMS antechamber walls and the inclusion of MAVEN measurements made during DD campaigns are required to pin down the vertical trend of ionization efficiency at low altitudes unambiguously.

The diurnal variations of the $\mathrm{CO}_{2}$ (blue) and $\mathrm{O}$ (red) ionization efficiencies are presented in Figure 3 (top). For each nominal MAVEN orbit, the median $\mathrm{CO}_{2}$ and $\mathrm{O}$ ionization efficiencies, as well as the respective median SZA, are evaluated. To be conservative, only the data acquired above $170 \mathrm{~km}$ are included where no vertical trends are revealed by the data. We then sort all orbits in increasing order of SZA and divide the entire data set into 12 consecutive bins, each containing an identical number of orbits from which the median $\mathrm{CO}_{2}$ and $\mathrm{O}$ ionization efficiencies are obtained. The figure does not reveal any substantial diurnal variation, except for a trend of reduced ionization efficiency approaching the terminator, but such a trend is insignificant in view of the large scatterings 


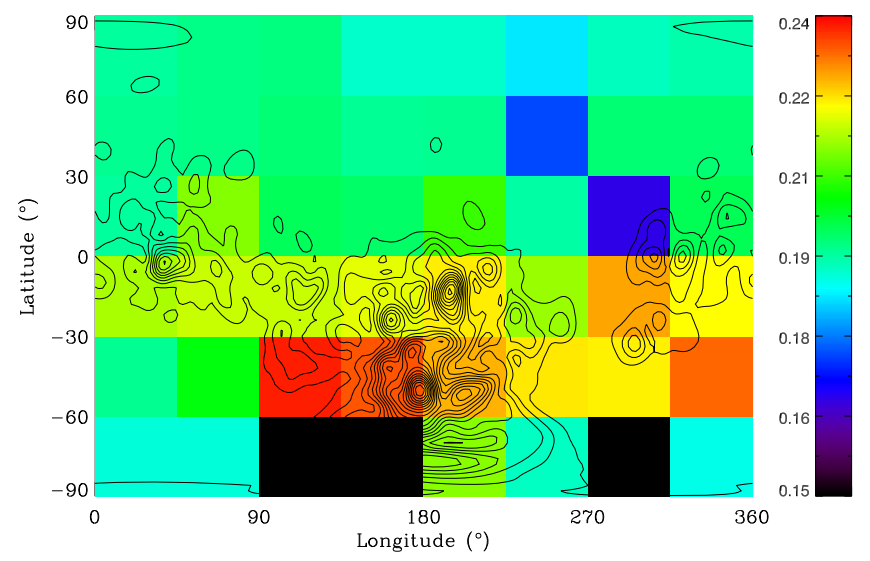

Figure 4. Distribution of $\mathrm{CO}_{2}$ ionization efficiency at the top of the dayside Martian upper atmosphere across the surface of Mars. The crustal magnetic field map of Langlais et al. (2004), appropriate for an altitude of $400 \mathrm{~km}$, is superimposed for comparison. The figure reveals an evident connection between regions with strong crustal fields and regions with high ionization efficiencies.

within the predefined SZA bins. This observation is in agreement with the model prediction of Nicholson et al. (2009) at similar altitudes (see their Figure 6). Meanwhile, the same authors found no solar cycle variation in ionization efficiency, a conclusion that is also confirmed by our analysis here as demonstrated in Figure 3 (bottom). We caution that only a portion of the full solar cycle has been sampled by $M A V E N$ up to now, over a range of input solar EUV/X-ray energy flux of $0.8-2 \mathrm{erg} \mathrm{cm}^{-2} \mathrm{~s}^{-1}$ that characterizes low to moderate solar activities.

Finally, it is instructive to evaluate the impact of crustal magnetic fields. Here we divide the entire surface of Mars into 48 sectors, each with a size of $30^{\circ}$ in latitude and $45^{\circ}$ in longitude. The respective median $\mathrm{CO}_{2}$ ionization efficiencies above $170 \mathrm{~km}$ are color coded in Figure 4, blue for low efficiencies and red for high efficiencies. Such a procedure is validated by the lack of clear diurnal and solar cycle variations as described above. The three sectors near the south pole color coded by black contain insufficient numbers of individual measurements, and are thus excluded from subsequent analysis. The crustal magnetic field map of Langlais et al. (2004), appropriate for an altitude of $400 \mathrm{~km}$, is superimposed for comparison. The figure reveals a tentative connection between regions with strong crustal fields and regions with high ionization efficiencies. In particular, the two sectors with the highest $\mathrm{CO}_{2}$ ionization efficiencies are nearly collocated with the strong crustal magnetic anomaly near Terra Cimmeria (Langlais et al. 2004). The distribution of $\mathrm{O}$ ionization efficiency (not shown in the figure) reveals a quite similar pattern.

\section{Discussions and Concluding Remarks}

Combining the NGIMS, EUVM, and SWEA measurements of neutral atmospheric density, solar EUV/X-ray flux, and differential photoelectron intensity made during 240 nominal $M A V E N$ orbits, we calculate the ionization efficiency, defined as the ratio of the secondary ionization rate to the primary ionization rate, in the dayside Martian upper atmosphere under a range of solar illumination conditions. The photoelectron energy spectra are corrected for spacecraft charging using the LPW potentials, and a portion of the spectra showing strong fluctuations at the top of the atmosphere, likely indicative of significant energy input via SW electron precipitation, are excluded. This ensures a clean selection of orbits where secondary ionization is predominantly caused by photoelectrons. The data from the dayside DD campaign on 2015 April 17-22, as well as the outbound data from all nominal orbits, are excluded to reduce the effect of NGIMS wall chemistry.

Our analysis reveals that both the $\mathrm{CO}_{2}$ and $\mathrm{O}$ ionization efficiencies remain constant over the altitude range of $160-250 \mathrm{~km}$. No substantial diurnal and solar cycle variations are suggested by the data, except for an insignificant trend of reduced ionization efficiency approaching the terminator. At the top of the atmosphere, the median ionization efficiencies are $0.19 \pm 0.03$ for $\mathrm{CO}_{2}$ and $0.27 \pm 0.04$ for $\mathrm{O}$, respectively, in fair agreement with various model results covering a range of solar irradiance levels from low to high solar activities and a range of solar illumination angles from subsolar to nearterminator (e.g., Fox \& Yeager 2006; Haider et al. 2006; Nicholson et al. 2009). These values are useful for fast calculations of the total ionization rate in the dayside Martian upper atmosphere, without the need to construct photoelectron transport models. A tentative trend of enhanced ionization efficiency is observed near the periapsis of nominal MAVEN orbits. The inclusion of extra data gathered during dayside DD campaigns, along with a rigorous treatment of NGIMS wall chemistry, is required to pin down the vertical trend at low altitudes unambiguously. Our analysis also reveals a connection between regions with strong crustal magnetic fields and regions with relatively high ionization efficiencies.

The overall lack of substantial vertical, diurnal, and solar cycle variations in ionization efficiency, as well as the difference between $\mathrm{CO}_{2}$ and $\mathrm{O}$ could be interpreted in a simple way, suggesting that, as a first approximation, the ionization efficiency relies on a comparison between relevant cross sections. Specifically, we assume, without loss of generality, a monochromatic solar flux at $36 \mathrm{~nm}$, producing photoelectrons at $20 \mathrm{eV}$, which is also the weighted mean photoelectron energy in the dayside Martian upper atmosphere well above the ionospheric peak (e.g., Fox \& Yeager 2006). Assuming local energy deposition, the respective photoelectron flux is controlled by a balance between production, which is primary ionization, and loss, which is downward degradation via inelastic collisions with ambient $\mathrm{CO}_{2}$. Equating the production and loss rates, the photoelectron flux is found to be identical to the solar flux at $36 \mathrm{~nm}$ multiplied by 0.036 , which is the ratio between the $\mathrm{CO}_{2}$ photoionization cross section, $5.7 \times 10^{-18} \mathrm{~cm}^{2}$ at $36 \mathrm{~nm}$, and the $\mathrm{CO}_{2}$ electron impact cross section summed over all channels leading to excitation and ionization, $1.6 \times 10^{-16} \mathrm{~cm}^{2}$ at $20 \mathrm{eV}$. Both the primary and secondary ionization rates are proportional to the neutral density, the flux of ionizing particles (photons or photoelectrons), as well as the respective ionization cross section. Accordingly, the $\mathrm{CO}_{2}$ ionization efficiency is equal to the ratio of 6.3 between the $\mathrm{CO}_{2}$ electron impact ionization cross section of $3.6 \times 10^{-17} \mathrm{~cm}^{2}$ at $20 \mathrm{eV}$ and the $\mathrm{CO}_{2}$ photoionization cross section of $5.7 \times 10^{-18} \mathrm{~cm}^{2}$ at $36 \mathrm{~nm}$, multiplied by 0.036 quoted above. This gives a $\mathrm{CO}_{2}$ ionization efficiency of 0.2 . Similarly, the ratio between the $\mathrm{O}$ electron impact ionization cross section of $2.8 \times 10^{-17} \mathrm{~cm}^{2}$ and the $\mathrm{O}$ photoionization cross section of $2.6 \times 10^{-18} \mathrm{~cm}^{2}$ is 11 , leading to an $\mathrm{O}$ ionization efficiency of 0.4 when multiplied by 0.036 . 
These ionization efficiencies are in reasonable agreement with the MAVEN-based values.

The above crude estimate is complicated by (1) multiple ionization caused by atmospheric absorption of solar X-ray photons (e.g., Wedlund et al. 2011) and (2) the dominance of photoelectron transport over local energy degradation (e.g., Cui et al. 2011). The former leads to an ionization efficiency that increases sharply near or below the ionospheric peak where the optical depth for solar X-ray photons approaches unity (Fox \& Yeager 2006). This likely accounts for the apparent enhancement in ionization efficiency near the periapsis of nominal $M A V E N$ orbits. The latter should occur over the entire altitude range of interest here with the bottomside roughly coincident with the typical photoelectron exobase at around $160 \mathrm{~km}(\mathrm{Xu}$ et al. 2016). Transport serves as an additional loss mechanism, which reduces the secondary ionization rate and accounts for the modest difference between the MAVEN-based ionization efficiencies and those obtained by comparing cross sections only, especially for $\mathrm{O}$. It is also interesting to note that the cross-terminator flow, a special pattern of ion transport that helps to maintain the nightside ionosphere of Mars (e.g., Cui et al. 2015), tends to reduce the ionization efficiency at high SZA. This is likely responsible for the reduced ionization efficiency near the terminator, as is marginally seen in Figure 3 (top).

The apparent connection between regions with strong magnetic field strength and regions with high ionization efficiency, as revealed by the MAVEN data, is instructive. One possible interpretation is the trapping of in-situ produced photoelectrons by closed magnetic field lines typically found over strong crustal magnetic anomalies (e.g., Brain et al. 2007). Since photoelectrons also play a crucial role in the local energy balance of both neutrals and thermal electrons, we expect enhanced neutral and electron temperatures encountered near magnetic anomalies as well. The observation of enhanced neutral temperature was reported by Cui et al. (2018) with the aid of the NGIMS data acquired during several DD campaigns (see also Leblanc et al. 2006), but these authors argued that photoelectron trapping was unlikely to be a viable mechanism since the difference in photoelectron impact heating between regions with and without strong crustal magnetic fields was far insufficient to account for the difference in neutral temperature. Meanwhile, Flynn et al. (2017) showed that regions over magnetic anomalies featured low electron temperatures, in contrast to our ideal expectation. According to Xu et al. (2017), the magnetic field configuration throughout the entire atmospheric regions of interest here is dominated by closed field lines (see their Figure 8), indicating that photoelectrons are always trapped at these altitudes irrespective of the magnetic field strength. However, the same authors concluded that the field lines in regions with strong magnetic fields were more vertical as compared to regions with weak fields (see their Figure 12). Therefore, it is more likely a higher tendency for vertical photoelectron transport that is responsible for the observed enhancement in ionization efficiency near strong crustal anomalies. For comparison, a higher tendency for vertical diffusion is thought to contribute to the enhanced thermal electron content in the Martian upper atmosphere also observed near crustal anomalies (Ness et al. 2000; Nielsen et al. 2007; Safaeinili et al. 2007), as supported by the model calculations of Matta et al. (2015). Clearly, the construction of realistic photoelectron transport models with properly imposed ambient magnetic field topology is required to interpret unambiguously the observed impact of crustal fields on ionization efficiency.

J.C. and Y.W. acknowledge support from the National Science Foundation of China through grants 41525015, 41774186, and 41525016. J.C. is also supported by the Science and Technology Development Fund of Macau SAR through grants $039 / 2013 / \mathrm{A} 2$ and $119 / 2017 / A 3$. S.X. is supported by the NASA MAVEN project through the Mars Exploration Program. A.W. acknowledges support from the STFC consolidated grants to UCL-MSSL ST/K000977/1 and ST/N000722/1. T.A.N. is supported by an appointment to the NASA Postdoctoral Program administered by Oak Ridge Associated Universities and Universities Space Research Association through a contract with NASA. The authors thank the anonymous referee for constructive comments, which have greatly improved the manuscript.

\section{ORCID iDs}

\section{J. Cui (iD https://orcid.org/0000-0002-4721-8184}

\section{References}

Acuna, M. H., Connerney, J. E. P., Ness, N. F., et al. 1999, Sci, 284, 790 Andersson, L., Ergun, R. E., Delory, G. T., et al. 2015, SSRv, 195, 173 Bhardwaj, A., \& Jain, S. K. 2009, JGRA, 114, A11309

Brain, D. A., Lillis, R. J., Mitchell, D. L., Halekas, J. S., \& Lin, R. P. 2007, JGRA, 112, A09201

Cui, J., Galand, M., Coates, A. J., Zhang, T. L., \& Müller-Wodarg, I. C. F. 2011, JGRA, 116, A04321

Cui, J., Galand, M., Yelle, R. V., Wei, Y., \& Zhang, S.-J. 2015, JGRA, 120,2333

Cui, J., Lian, Y., \& Müller-Wodarg, I. C. F. 2013, GeoRL, 40, 43

Cui, J., Yelle, R. V., Li, T., Snowden, D. S., \& Müller-Wodarg, I. C. F. 2014, JGRA, 119, 490

Cui, J., Yelle, R. V., Vuitton, V., et al. 2009, Icar, 200, 581

Cui, J., Yelle, R. V., Zhao, L.-L., et al. 2018, ApJL, 853, L33

Curry, S. M., Luhmann, J. G., Ma, Y. J., et al. 2015, GeoRL, 42, 9095

Dong, C., Ma, Y., Bougher, S. W., et al. 2015, GeoRL, 42, 9103

England, S. L., Liu, G., Yiğit, E., et al. 2017, JGRA, 122, 2310

Eparvier, F. G., Chamberlin, P. C., Woods, T. N., \& Thiemann, E. M. B. 2015, SSRv, 195, 293

Flynn, C. L., Vogt, M. F., Withers, P., et al. 2017, GeoRL, 44, 10812

Fox, J. L., \& Yeager, K. E. 2006, JGRA, 111, A10309

Galand, M., Moore, L., Charnay, B., Mueller-Wodarg, I., \& Mendillo, M. 2009, JGRA, 114, A06313

Haider, S. A., Seth, S. P., Choksi, V. R., \& Oyama, K. I. 2006, Icar, 185, 102 Ionov, D. E., Bisikalo, D. V., Shematovich, V. I., \& Huber, B. 2014, SoSyR, 48, 105

Jakosky, B. M., Grebowsky, J. M., Luhmann, J. G., \& Brain, D. A. 2015 , GeoRL, 42, 8791

Langlais, B., Purucker, M. E., \& Mandea, M. 2004, JGRE, 109, E02008

Leblanc, F., Chaufray, J. Y., Lilensten, J., Witasse, O., \& Bertaux, J.-L. 2006, JGRE, 111, E09S11

Lilensten, J., Kofman, W., Wisemberg, J., Oran, E. S., \& DeVore, C. R. 1989, AnGeo, 7, 83

Lilensten, J., Simon, C., Witasse, O., et al. 2005, Icar, 174, 285

Mahaffy, P. R., Benna, M., Elrod, M., et al. 2015a, GeoRL, 42, 8951

Mahaffy, P. R., Benna, M., King, T., et al. 2015b, SSRv, 195, 49

Matta, M., Mendillo, M., Withers, P., \& Morgan, D. 2015, JGRA, 120, 766

Mendillo, M., Narvaez, C., Vogt, M. F., et al. 2017, JGRA, 122, 9670

Mitchell, D. L., Mazelle, C., Sauvaud, J.-A., et al. 2016, SSRv, 200, 495

Ness, N. F., Acuña, M. H., Connerney, J. E. P., et al. 2000, JGR, 105, 15991

Nicholson, W. P., Gronoff, G., Lilensten, J., Aylward, A. D., \& Simon, C. 2009, MNRAS, 400, 369

Nielsen, E., Fraenz, M., Zou, H., et al. 2007, P\&SS, 55, 2164

Peterson, W. K., Thiemann, E. M. B., Eparvier, F. G., et al. 2016, JGRA, 121,8859

Richards, P. G., \& Torr, D. G. 1988, JGR, 93, 4060

Safaeinili, A., Kofman, W., Mouginot, J., et al. 2007, GeoRL, 34, L23204 
Sakai, S., Rahmati, A., Mitchell, D. L., et al. 2015, GeoRL, 42, 8894

Smith, F. L., III, \& Smith, C. 1972, JGR, 77, 3592

Thiemann, E. M. B., Chamberlin, P. C., Eparvier, F. G., et al. 2017, JGRA, 122,2748

Wedlund, C. S., Gronoff, G., Lilensten, J., Ménager, H., \& Barthélemy, M. 2011, AnGeo, 29, 187
Withers, P. 2009, AdSpR, 44, 277

Xu, S., Liemohn, M., Bougher, S., \& Mitchell, D. 2016, JGRA, 121, 3767

Xu, S., \& Liemohn, M. W. 2015, E\&SS, 2, 47

Xu, S., Liemohn, M. W., Peterson, W. K., Fontenla, J., \& Chamberlin, P. 2015 , P\&SS, 119, 62

Xu, S., Mitchell, D., Liemohn, M., et al. 2017, JGRA, 122, 1831 